\title{
Transcription factor c-Rel plays a crucial role in driving anti-CD40-mediated innate colitis
}

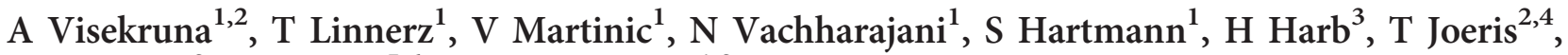 \\ PI Pfefferle ${ }^{2}$, MJ Hofer ${ }^{5,6}$ and U Steinhoff ${ }^{1,2}$
}

Genetic and environmental factors, including the commensal microbiota, have a crucial role in the development of inflammatory bowel disease. Aberrant activation of the transcription factor NF-кB is associated with chronic intestinal inflammation in mice and humans. Recently, an emerging family of innate lymphoid cells (ILCs) has been identified at mucosal sites contributing to the maintenance of gut homeostasis and intestinal immunopathology. Here, we show that the NF-кB protein c-Rel regulates the inflammatory potential of colonic IFN- $\gamma{ }^{+}$Thy $1^{+}$ILCs to induce anti-CD40mediated colitis in rag1 ${ }^{-/-}$mice. Stimulation of dendritic cells (DCs) with anti-CD40 or CD40L led to translocation of C-Rel into the nucleus resulting in induction of expression of interleukin-12 (IL-12) and IL-23, key regulators of innate cell-induced colitis. While c-Rel deficiency completely abrogated anti-CD40-induced colitis, adoptively transferred wild-type DCs were able to induce pronounced colonic inflammation in rag $1^{-1}{ }^{-1} \mathrm{rl}^{-1-}$ mice. In summary, these results suggest that the expression of c-Rel in DCs is essential for initiating anti-CD40-mediated intestinal pathogenesis.

\section{INTRODUCTION}

Chronic inflammation triggered by microbiota-dependent innate and adaptive immune responses is the main driving force in the development of inflammatory bowel disease. ${ }^{1}$ The role of NF- $\kappa \mathrm{B}$ as a crucial factor contributing to the initiation and maintenance of chronic inflammation has been wellknown. ${ }^{2,3}$ The five NF- $\kappa$ B family members, p65 (RelA), RelB,

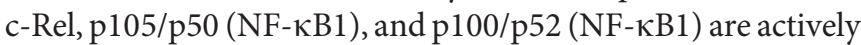
involved in central cellular processes such as proliferation, differentiation, and survival. ${ }^{4}$ In unstimulated cells, NF- $\kappa \mathrm{B}$ proteins pre-exist as inactive dimers associated with one of the inhibitory $\mathrm{I} \kappa \mathrm{B}$ proteins such as $\mathrm{I} \kappa \mathrm{B} \alpha$, I $\mathrm{I} B \beta$, and $\mathrm{I} \kappa \mathrm{B} \varepsilon{ }^{5}$ Stimulation of cells with appropriate stimuli leads to translocation of active NF- $\kappa \mathrm{B}$ dimers into the nucleus. ${ }^{6}$ The signaling to $\mathrm{NF}-\kappa \mathrm{B}$ is generally considered to occur through either degradation of cytoplasmic I $\mathrm{KB}$ proteins (classical pathway) or through processing of p100 to form p52 (alternative pathway). The third signaling cascade, so-called p105 pathway, shares some similarities with classical NF- $\kappa \mathrm{B}$ pathway and is responsible for rapid degradation of p105 and release of active NF- $\kappa \mathrm{B}$ subunits. ${ }^{7}$

During intestinal inflammation, $N F-\kappa B$ proteins regulate induction of the transcription of genes encoding various proinflammatory cytokines, chemokines, and adhesions molecules thus augmenting chronic inflammatory responses. ${ }^{8,9}$ The $\mathrm{NF}-\kappa \mathrm{B}$ family member $\mathrm{c}$-Rel has an important role in the innate and adaptive immunity by regulating the function of antigen presenting cells (APCs) and lymphocytes. ${ }^{10-12}$ Innate lymphoid cells (ILCs) are an emerging immune cell population involved in the mucosal tissue homeostasis and onset of inflammation in the intestine. ${ }^{13,14}$ Mirroring the complexity of the distinct T-helper (Th) subpopulations that secrete characteristic signature cytokines, an expanding family of ILCs that are capable of producing many Th cell-associated cytokines has been recently described. ${ }^{15,16}$ ILCs are heterogeneous consisting of at least three subpopulations, ILC1 secreting interferon- $\gamma$ (IFN- $\gamma$ ), ILC2 expressing GATA-3 and producing Th2 cellrelated cytokines and the ILC3 population which expresses

\footnotetext{
${ }^{1}$ Institute for Medical Microbiology and Hygiene, Philipps University of Marburg, Marburg, Germany. ${ }^{2}$ Max Planck Institute for Infection Biology, Department of Immunology, Berlin, Germany. ${ }^{3}$ Institute of Laboratory Medicine and Pathobiochemistry, Molecular Diagnostics, Philipps University of Marburg, Marburg, Germany. ${ }^{4}$ mmunology Section, Department of Experimental Medical Science, Lund University, Lund, Sweden. ${ }^{5}$ Department of Neuropathology, Philipps University of Marburg, Marburg, Germany and ${ }^{6}$ School of Molecular Bioscience, The University of Sydney, Darlington, New South Wales, Australia. Correspondence: U Steinhoff or A Visekruna (ulrich.steinhoff@staff. uni-marburg.de or alexander.visekruna@staff.uni-marburg.de)
} 
ROR $\gamma$ t and secretes Th17-associated cytokines interleukin-17 (IL-17) and IL-22. ${ }^{17}$ Recently, several reports demonstrated an essential role for ILCs during development of gut inflammation in mouse models of colitis. ${ }^{13,18}$ Mice deficient for T and B cells injected with anti-CD40 monoclonal antibody (mAb) developed colitis and rapid weight loss. ${ }^{19}$ Depletion of Thy $1^{+}$ILCs in $r a g 1^{-/-}$mice was shown to completely abrogate anti-CD40 $\mathrm{mAb}$-induced gut inflammation demonstrating a key role for this immune cell population in mediating intestinal immunopathology in the absence of $\mathrm{T}$ cells. ${ }^{13}$

In contrast to the well-defined function of c-Rel in T-cellmediated immunity, the role of this transcription factor in promoting anti-CD40-induced innate intestinal inflammation has not been addressed yet. We here described that the absence of c-Rel protects rag1 ${ }^{-/-}$mice from innate cell-driven colitis. Moreover, we provide evidence that CD40 stimulation leads to activation of c-Rel in dendritic cells (DCs), which in turn triggers Thy ${ }^{+}$ILCs to produce IFN- $\gamma$ and promote intestinal inflammation.

\section{RESULTS \\ CD40 ligation induces c-Rel activation in DCs}

Members of NF- $\kappa$ B family of transcription factors have a central role in the initiation of chronic inflammation, however their impact on anti-CD40-induced innate colitis is not known yet. To analyze the functional role of c-Rel in promoting antiCD40-induced intestinal inflammation, we first examined whether CD40L and anti-CD40 stimulation of DCs led to activation of this transcription factor. So far, CD40 has been reported to strongly activate the processing of NF- $\kappa \mathrm{B} 2 \mathrm{p} 100$ to $\mathrm{p} 52$, thus promoting the nuclear translocation of p52-RelB dimers in primary B cells. ${ }^{20,21}$ In accordance with previous data, the electrophoretic mobility shift assay (EMSA) experiments clearly demonstrated that both, CD40L and anti-CD40 strongly activated NF- $\kappa$ B in bone marrow DCs (BMDCs; Figure 1a). However, not only activation of the alternative NF- $\kappa B$ pathway but also CD40-induced nuclear translocation of c-Rel was observed after CD40L or anti-CD40 stimulation for $2 \mathrm{~h}$ (Figure 1b, data not shown). To quantify transcriptional a

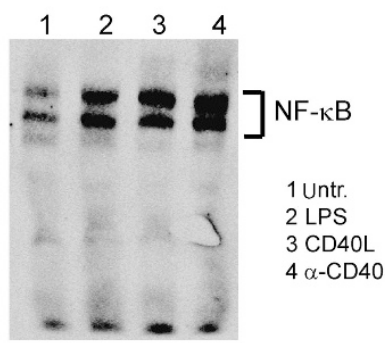

C

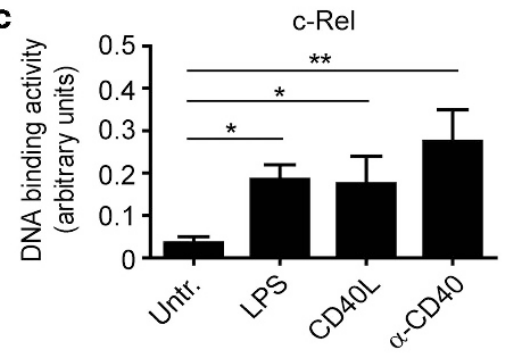

e

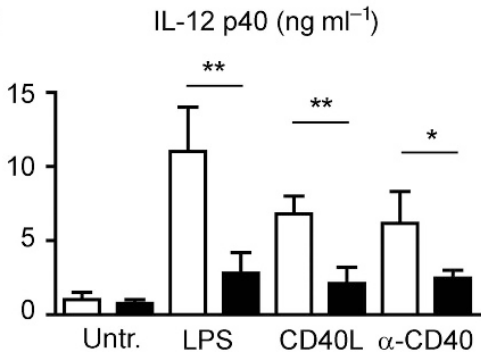

b

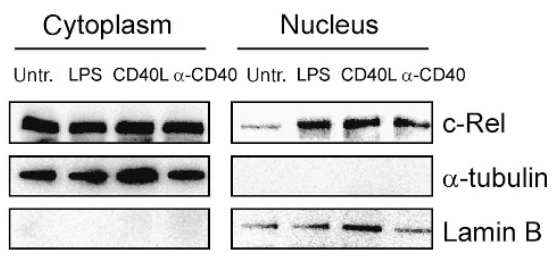

d

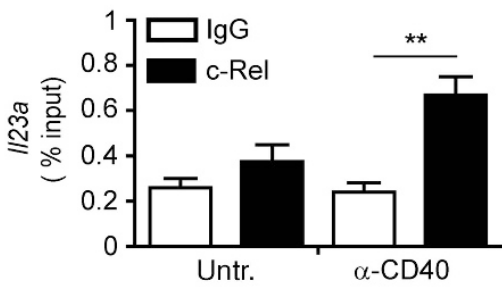

IL-23 $\left(\mathrm{pg} \mathrm{ml}^{-1}\right)$

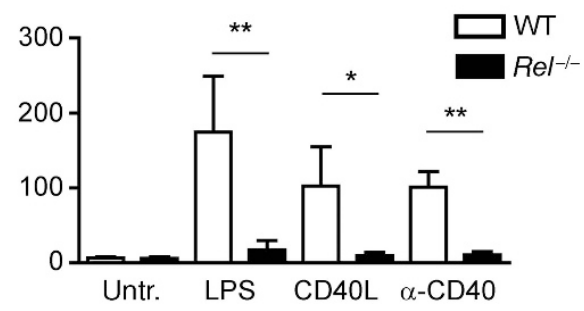

Figure 1 Stimulation of bone marrow dendritic cells (BMDCs) by anti-CD40 and CD40L results in the translocation of c-Rel into the nucleus. (a) BMDCs generated from C57BL/6 mice were stimulated for $2 \mathrm{~h}$ with lipopolysaccharide (LPS) $\left(1 \mu \mathrm{g} \mathrm{ml}^{-1}\right), \mathrm{CD} 40 \mathrm{~L}\left(1 \mu \mathrm{g} \mathrm{ml}^{-1}\right)$ and anti-CD40 (10 $\left.\mu \mathrm{g} \mathrm{ml}{ }^{-1}\right)$. Nuclear extracts were analyzed for NF- $\mathrm{kB}$ binding by electrophoretic mobility shift assay (EMSA). Control EMSA reactions were performed in the absence of stimulations. (b) Nuclear and cytoplasmic extracts of WT BMDCs were treated with or without LPS, CD40L, or anti-CD40 for $2 \mathrm{~h}$. The amounts of c-Rel and $\beta$-actin were examined by immunoblotting analysis. (c) Binding of c-Rel to an NF-kB-binding oligonucleotide sequence after stimulation of wild-type (WT) BMDCs with LPS, CD40L, or anti-CD40 was analyzed by ELISA-based assay (Active Motif). Data represent mean \pm s.e.m. ( $n=4)$. (d) WT BMDCs were treated with $10 \mu \mathrm{g} \mathrm{ml}^{-1}$ anti-CD40 for $2 \mathrm{~h}$. Nuclear extracts were prepared and analysis of c-Rel binding to the $\kappa 1$ site of $p 19$ promotor was performed by Chromatin Immunoprecipitation. Three independent experiments were carried out. Data represent mean \pm s.e.m.. (e) DCs from WT and c-Rel-deficient mice were either left untreated or stimulated with LPS, CD40L, or anti-CD40. After $24 \mathrm{~h}$, supernatants were analyzed for interleukin-12 (IL-12) p40 and IL-23 secretion. Data from two independent experiments were pooled. Data represent mean \pm s.e.m. ${ }^{*} P=0.01-0.05,{ }^{\star \star} P=0.001-0.01$. 
activity of c-Rel, binding to the NF- $\kappa \mathrm{B}$ consensus site was measured following stimulation of BMDCs with lipopolysaccharide (LPS), CD40L, and anti-CD40. Similar to LPS stimulation, the c-Rel DNA-binding activity was significantly increased after stimulation of BMDCs with CD40L or antiCD40 (Figure 1c). Thus, CD40 stimulates not only translocation of p52-RelB but also that of c-Rel-containing dimers. Further, in line with previous findings showing that c-Rel is essential for LPS-induced IL-12 p40 and IL-23 p19 expression, ${ }^{22,23}$ chromatin immunoprecipitation analysis revealed that stimulation of BMDCs with anti-CD40 resulted in binding of c-Rel to the IL-23 p19 promotor sequence (Figure 1d). Finally, treatment with both, CD40L and anti-CD40 led to induction of secretion of IL-12 p40 and IL-23 in wild-type (WT) but not in c-Rel-deficient BMDCs (Figure 1e). LPSinduced cell surface expression of CD40 was described to be diminished in $\mathrm{p50}^{-/-} \mathrm{rel}^{-/-}$DCs compared with WT cells. ${ }^{11}$ As downregulation of CD40 in $\mathrm{rel}^{-/-}$cells might be responsible for reduced cytokine secretion, WT and c-Rel-deficient DCs were stimulated with LPS, CD40L, and anti-CD40, and CD40 expression was examined by flow cytometry analysis. No significant reduction of CD40 or MHC II expression on $\mathrm{rel}^{-/-}$ cells was observed as compared with WT BMDCs. Similar data were obtained when proliferation of c-Rel-deficient B cells was measured following CD40L stimulation (Supplementary Figure S1 online). We hypothesized that CD40-mediated activation of c-Rel in DCs might be linked with IL-12/IL-23regulated inflammatory functions of ILCs during the development of intestinal pathology.

\section{c-Rel signaling is required for anti-CD40-induced intestinal inflammation}

To examine if c-Rel is required for the development of antiCD40-induced colitis, we crossed c-Rel-deficient mice with rag1 ${ }^{-/-}$animals and treated both $\mathrm{ragl}^{-I^{-}}$and $\mathrm{ragl}^{-{ }^{-}}$ $\mathrm{rel}^{-1-}$ mice with anti-CD40 $\mathrm{mAb}$. rag1 ${ }^{-1-}$ mice injected intraperitonealy (IP) with $200 \mu \mathrm{g}$ of anti-CD40 mAb, clone FGK45, have been shown to develop histological signs of colitis within 7 days. $^{19}$ Furthermore, depletion of Thy ${ }^{+}$ILCs protected $\mathrm{rag1}^{-1-}$ animals from anti-CD40-induced intestinal inflammation. ${ }^{13}$ To determine whether these findings are specific for FGK45 or apply also to other agonistic anti-CD40 Abs, we injected clone 1C10, which we used for in vitro stimulation of BMDCs (Figure 1). Similar to FGK45-mediated colitis, IP injection of $100 \mu \mathrm{g}$ of $1 \mathrm{C} 10$ led to development of colitis in rag1 ${ }^{-/-}$mice with macroscopic and histological signs of colon pathology. While IP administration of 1C10 to rag1 ${ }^{-/-}$animals triggered potent systemic and gut inflammation with loss of weight, increased amounts of serum cytokine concentrations, gut edema, epithelial hyperplasia, and leukocyte infiltration into colonic lamina propria, $\mathrm{ragl}^{-/-} \mathrm{rel}^{-/-}$ mice developed neither signs of intestinal inflammation and nor systemic immunopathology (Figure 2a-c, data not shown). Furthermore, analysis of IL-12 and IL-23 gene expression upon induction of anti-CD40-mediated colitis showed that colonic tissue from rag1 ${ }^{-/-}$mice expressed significantly more IL-12 p40, IL-12 p35, and IL-23 p19 messenger RNA than colonic rag1 ${ }^{-1-} \mathrm{rel}^{-1-}$ cells (Figure 3a). Similarly, secretion of colonic IL-23 after anti-CD40 treatment was much stronger in rag1 ${ }^{-1-}$ mice than in rag1 ${ }^{-1-} \mathrm{rel}^{-/-}$animals. On the other hand, we found no differences in the colonic messenger RNA expression of IL-23R between rag1 ${ }^{-/-}$and $r a g 1^{-1-}$ $\mathrm{rel}^{-/-}$mice (Figure 3b and c). Taken together, our results indicate that the transcription factor c-Rel has a crucial role in the development of anti-CD40-induced innate colitis but seems not to impact on expression of IL-23R in colonic Thy $1^{+}$ILCs, which have been shown to be the only $\mathrm{IL}-23 \mathrm{R}^{+}$cell population in the colon of rag1 ${ }^{-/-}$mice. $^{24}$

\section{c-Rel drives IFN- $\gamma$-mediated intestinal pathology in rag1 $^{-/-}$mice}

Cytokine secretion by colonic lamina propria cells from antiCD40-treated mice was examined after overnight culture. Whereas colonic lamina propria cells from healthy mice secreted only marginal amounts of proinflammatory cytokines, CD40-stimulated rag1 $^{-1-}$ mice strongly elevated IFN- $\gamma$ and tumour-necrosis factor- $\alpha$ but not IL-17A production in the colon. In contrast, only moderate increase in secretion of inflammatory cytokines was observed in anti-CD40 $\mathrm{mAb}$ treated $\mathrm{ragl}^{-1-} \mathrm{rel}^{-1-}$ animals (Figure $4 \mathbf{a}-\mathbf{c}$ ). It has been suggested that IFN- $\gamma$-secreting Thy1 ${ }^{+}$ILCs are colitogenic cells following injection of anti-CD40 $\mathrm{mAb}$ into $\mathrm{rag}^{-1-}$
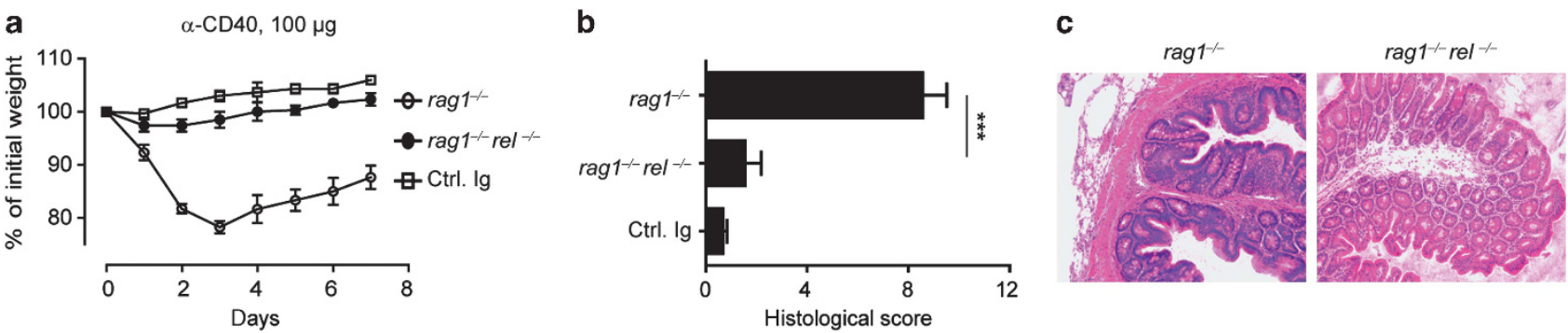

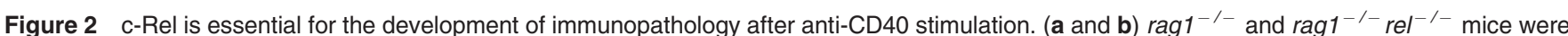
administered $100 \mu \mathrm{g}$ of anti-CD40 monoclonal antibody (mAb), clone1C10. Weight loss was assessed each day throughout the course of the experiment (a). At day 7 post anti-CD40 treatment, the histological score was determined (b). Data represent mean \pm s.e.m. of six mice per group. (c) Hematoxylin

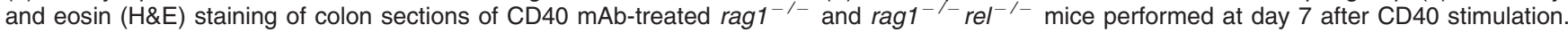
A representative of six mice per group is shown, original magnification $200 \times{ }^{* \star *} P<0.001$. 

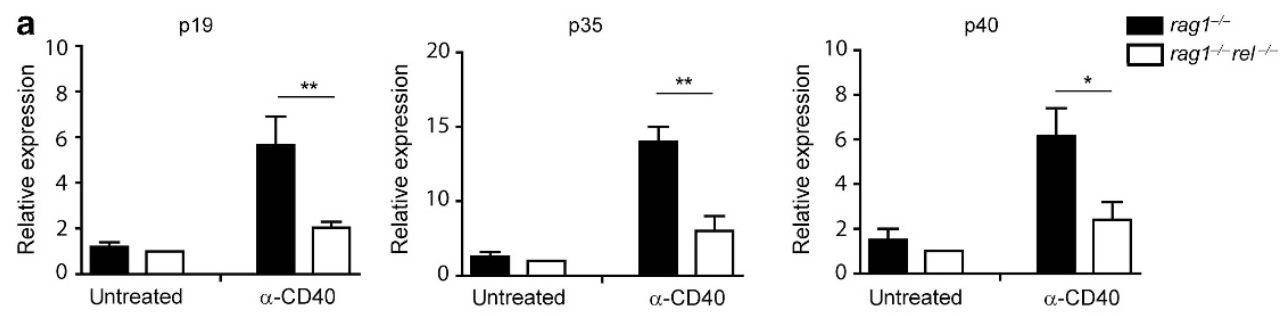

b
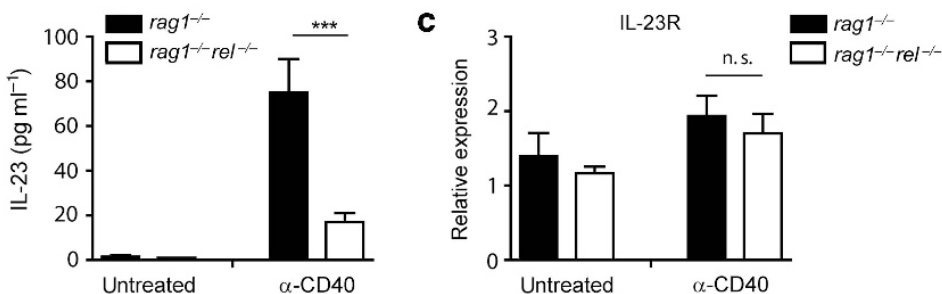

Figure 3 Induction of colonic interleukin-23 (IL-23) and IL-12 expression after in vivo treatment with anti-CD40 Ab. (a) rag1 $1^{-/-}$and rag $1^{-/-}$rel $^{-/-}$ mice were intraperitoneally (IP) injected with $100 \mu \mathrm{g}$ anti-CD40 monoclonal antibody (mAb) (1C10). At day 7 following stimulation with anti-CD40, the relative expression of IL-23p19, IL-12p35, and IL-12p40 messenger RNA (mRNA) was determined within the colons of rag1 ${ }^{-/-}$(filled bars) and rag $1^{-1-} \mathrm{rel}^{-1-}$ (open bars) mice. Data show mean \pm s.e.m. ( $n=5$ mice per group). (b) Induction of IL-23 protein expression was detected on day 7 after treatment of mice with anti-CD40 mAb. Colon pieces of anti-CD40-treated and untreated rag $1^{-1-}$ and rag $1^{-1-} \mathrm{rel}^{{ }^{-1-}}$ mice were cultured for $24 \mathrm{~h}$ and secretion of total IL-23 protein was measured by ELISA using both anti-p40 and anti-p19 antibodies. Data are mean \pm s.e.m. ( $n=5$ mice per group). (c) Expression of colonic IL-23R mRNA after in vivo treatment with anti-CD40 mAb. Data represent mean \pm s.e.m. $\left(n=5\right.$ mice per group). ${ }^{* * *} P<0.001$, ${ }^{\star *} P=0.001-0.01,{ }^{*} P=0.01-0.05$.

a

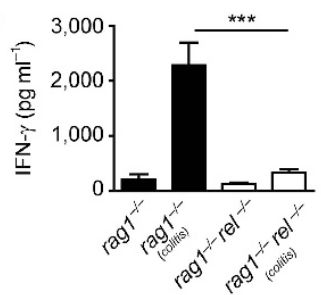

d

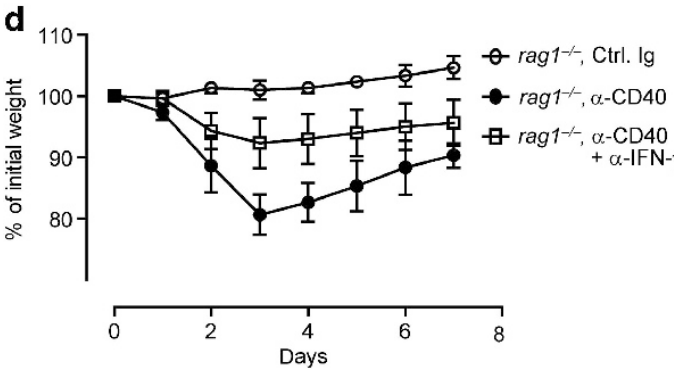

b

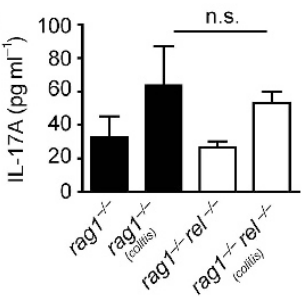

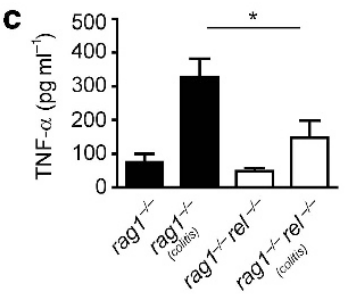

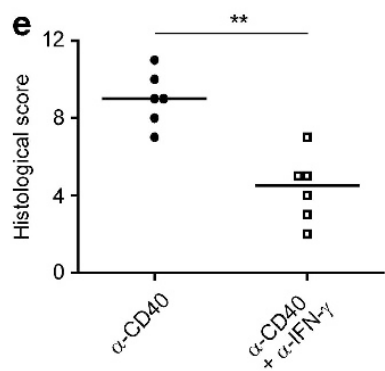

Figure 4 Production of proinflammatory cytokines within the colons of anti-CD40-treated rag $1^{-/-}$and rag $1^{-/-}$rel $^{-/-}$mice. (a) Cytokine secretion in overnight colon cultures at day 7 after induction of colitis by injecting $100 \mu \mathrm{g}$ anti-CD40 monoclonal antibody (mAb). Data represent mean $\pm \mathrm{s}$.e.m. $(n=5$ mice per group). (b) rag $1^{-1-}$ mice were administered $100 \mu \mathrm{g}$ of anti-CD40 Ab (1C10). Three milligrams of interferon- $\gamma$ (IFN- $\gamma$ ) blocking Ab (clone XMG1.2) were co-injected together with $1 \mathrm{C} 10$ and the weight loss was monitored for 7 days. Data represent mean \pm s.e.m. ( $n=6$ mice per group). (c) Histological colitis scores of rag $1^{-1-}$ mice treated with anti-CD40 and anti-IFN- $\gamma$ Abs. Data are mean \pm s.e.m. $\left(n=6\right.$ mice per group). ${ }^{* \star \star} P<0.001$, ${ }^{\star *} P=0.001-0.01,{ }^{*} P=0.01-0.05$.

mice. ${ }^{18}$ To test this, rag1 ${ }^{-/-}$mice were injected IP with anti$\mathrm{CD} 40 \mathrm{mAb}$ together with IFN- $\gamma$ blocking mAb. Indeed, blockade of IFN- $\gamma$ secretion led to significant reduction of weight loss and less pronounced intestinal pathology confirming that this cytokine has a colitis-promoting role after CD40 stimulation (Figure 4d and e). Accordingly, depletion of Thy $1^{+}$cells ameliorated colitis development in anti-CD40treated $\mathrm{ragl}^{-/-}$mice as previously shown (Figure 5a).
Following disease induction by the agonistic anti-CD40 Ab, Thy $1.2^{+}$cells were found to be the major source of IFN- $\gamma$ in the colonic lamina propria. Remarkably, while the onset of colitis in $r a g 1^{-/-}$mice correlated with the strong induction of IFN- $\gamma$ production by Thy $1.2^{+}$cells, anti-CD40 treatment of rag1 ${ }^{-/-} \mathrm{rel}^{-/-}$induced only moderately elevated IFN- $\gamma$ levels as compared with untreated mice (Figure $\mathbf{5 b}$ and $\mathbf{c}$ ). Colonic inflammation in $\mathrm{rag}^{-/-}$mice correlated with elevated 


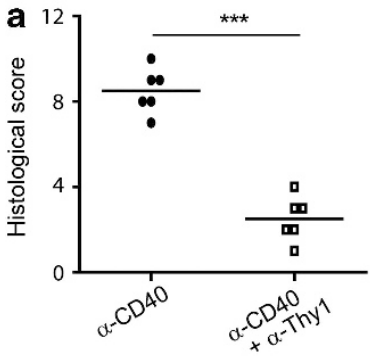

b
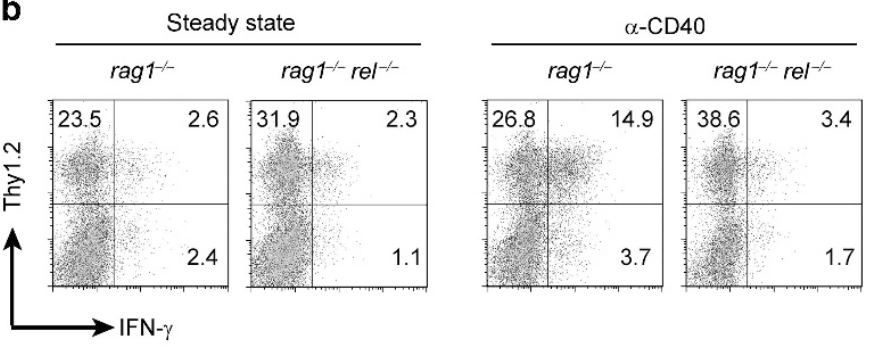

d

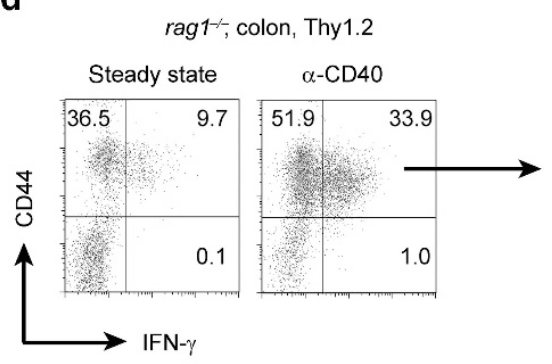

e
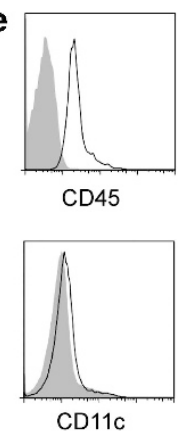

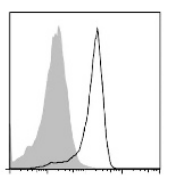

Sca-1

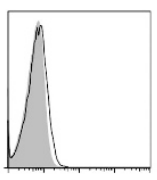

$\mathrm{CD} 11 \mathrm{~b}$

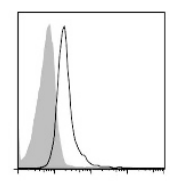

c-Kit

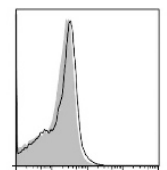

Gr-1
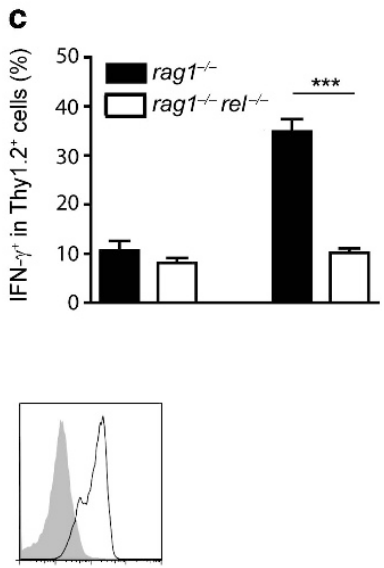

CD127

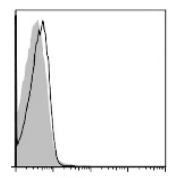

CD4

Figure 5 Characterization of colonic Thy $1^{+}$cells in rag $1^{-/-}$and $r a g 1^{-/-}$rel $^{-/-}$mice. (a) Histological colitis score of rag $1^{-/-}$mice following induction of anti-CD40-mediated colitis and depletion of Thy $1.2^{+}$cells with anti-Thy1 Ab. Data show mean \pm s.e.m. ( $n=5$ mice per group) (b) Intracellular production of interferon- $\gamma\left(\right.$ IFN- $\gamma$ ) by Thy $1.2^{\text {high }} \mathrm{LP}$ cells from anti-CD40-treated rag $1^{-1-}$ and rag $1^{-1-}$ rel $^{-/-}$mice measured by flow cytometry analysis at day 7 after colitis induction. Data are representative of three similar experiments. (c) Frequency of INF- $\gamma$-producing Thy $1.2^{+}$cells in colonic lamina propria following treatment of mice with anti-CD40 monoclonal antibody (mAb). Data show mean \pm s.e.m. ( $n=5$ mice per group). (d) Expression of CD44 on colonic Thy $1.2^{+}$cells after induction of anti-CD40-mediated colitis in rag1 ${ }^{-1-}$ mice. (e) On day 7 after induction of anti-CD40 colitis, colonic CD $44^{+}$IFN- $\gamma^{+}$Thy $1.2^{+}$cells from rag $1^{-/-}$mice were analyzed for the expression of CD45, Sca-1, C-Kit, CD127, Cd11c, CD11b, Gr-1, and CD4 by flow cytometry. $\mathbf{d}$ and $\mathbf{e}$ are representative of three similar experiments., ${ }^{\star \star \star} P<0.001$.

expression levels of CD44 within Thy1 ${ }^{+}$cell population. Interestingly, all IFN- $\gamma$-producing Thy $1.2^{+}$cells also expressed activation marker CD44 (Figure 5d). To better characterize the proinflammatory Thy $1.2^{+}$cell population, expression of various surface markers on IFN- $\gamma$-expressing Thy $1^{+}$cells was analyzed by flow cytometry after colitis induction in rag1 $1^{-1-}$ mice. No expression of CD11c, CD11b, Gr-1 as well as high expression levels of CD45 and common lymphoid progenitor markers Sca-1 and c-Kit suggest their hematopoetic Lin` ILC phenotype. Further examination revealed that CD4 is not expressed on these cells thus distinguishing them from $\mathrm{CD} 4{ }^{+}$LTi cells (Figure 5e). As intestinal NKp46 ${ }^{+} \mathrm{NK} 1.1^{+}$ cells comprise at least three distinct populations, conventional (c) natural killer (NK) cells, ILC1s, and NKp46 ${ }^{+} \mathrm{NK} 1.1^{+}$ ILC3s, all of them expressing IFN- $\gamma,{ }^{25}$ it is difficult to exactly define the expanded subset of IFN- $\gamma$-expressing Thy $1.2^{+}$cells based on the expression of NK1.1 and NKp46. However, because the majority of IFN- $\gamma^{+}$Thy $1.2^{+}$cells also expressed CD127, which is found on all ILC subpopulations apart from conventional NK cells, our data suggest that colitogenic IFN$\gamma^{+}$Thy1.2 $2^{+}$cell population might predominantly consists of ILC subsets (Figure 5e). Notably, IL-17A production was not induced within colonic Thy1.2 ${ }^{+}$ILCs after anti-CD40 mAb challenge (Supplementary Figure S2a), which is in line with a previous study describing an IL-17A-independent pathogenic role for ILCs in this colitis model. ${ }^{13}$
Transfer of WT DCs induces colitis and promotes IFN- $\gamma$ expression by colonic Thy $1.2^{+}$cell in rag1 ${ }^{-/-} \mathrm{rel}^{-/-}$mice As IP administration of anti-CD40 Ab did not lead to induction of CD40 on Thy $1.2^{+}$ILCs in rag1 $1^{-/-}$mice but strongly induced nuclear c-Rel activity in DCs (Figures $\mathbf{6 a}$ and $\mathbf{1 b}$ ), we assumed an indirect regulation of colitogenic Thy $1.2^{+}$cells by c-Rel derived from APCs. Further, total numbers of Thy $1.2^{+}$ ILCs in the colon of naive rag1 ${ }^{-/-}$and $\mathrm{ragl}^{-/-} \mathrm{rel}^{-/-}$mice were similar (Supplementary Figure S2b). To demonstrate the causative link between c-Rel expression in APCs and development of intestinal inflammation, LPS-stimulated WT or $\mathrm{rel}^{-/-}$BMDCs were injected IP into the rag1 ${ }^{-/-}$ $\mathrm{rel}^{-1-}$ mice prior to the treatment of these animals with anti-CD40 mAb. In contrast to $\mathrm{rel}^{-/-}$BMDCs, the transfer of WT BMDCs into ragl ${ }^{-/-} \mathrm{rel}^{-/-}$mice was sufficient to induce intestinal inflammation, loss of body weight, and an increase in IFN- $\gamma$ expression by colonic Thy $1.2^{+}$cells (Figure $\mathbf{6 b}-\mathbf{e}$ ). These results strongly suggest that c-Rel expression in inflammatory DCs is responsible for development of antiCD40-mediated intestinal inflammation.

Finally, we performed immunohistochemical staining of c-Rel expression in the inflamed gut of Crohn's disease patients. Significantly increased expression of c-Rel was observed in infiltrating inflammatory cells in patients with Crohn's disease patients as compared with the low expression of this transcription factor in the healthy colon. In particular, we found 

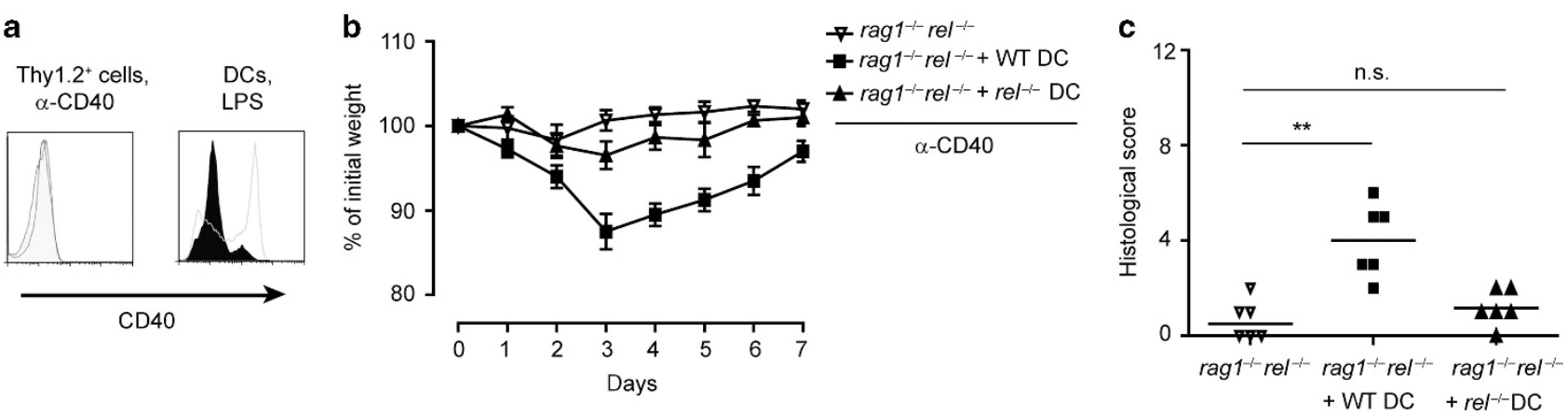

d

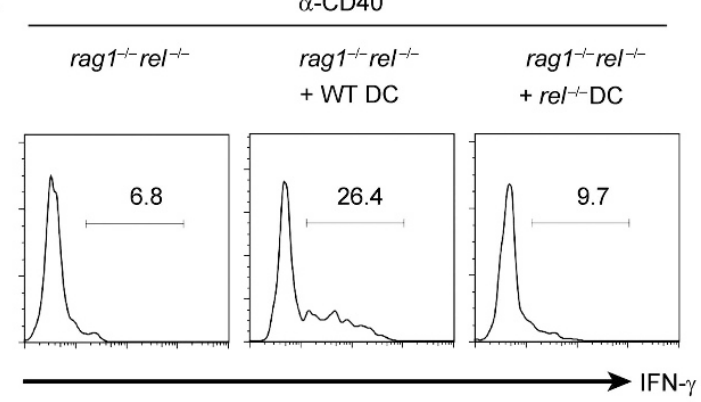

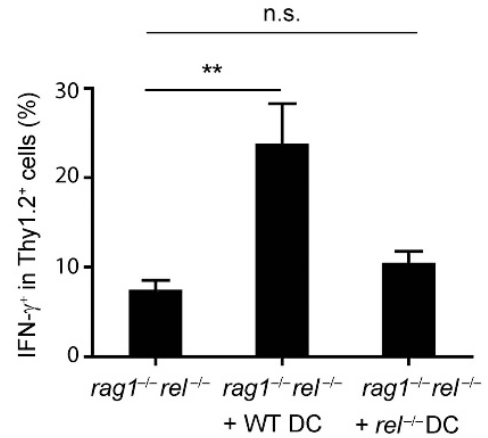

Figure 6 Transfer of wild-type (WT) DCs promotes the development of anti-CD40-induced colitis in rag1 ${ }^{-/-}$rel $^{-/-}$mice. (a) Surface expression of CD40 on colonic Thy $1^{\text {high }}$ LP cells at day 3 after induction of colitis by anti-CD40 in rag $1^{-1-}$ mice. As a positive control for CD40 expression, lipopolysaccharide (LPS)-stimulated bone marrow bone marrow dendritic cells (BMDCs) were used. CD40 expression levels were compared with isotype controls (gray shaded-Thy $1^{\text {high }}$ LP cells and black filled-BMDCs). (b and c) Measurement of weight loss and histological colitis score after transfer of LPS-stimulated WT or re1 $1^{-/-}$BMDCs into the rag1 ${ }^{-/} \mathrm{rel}^{-/-}$host and induction of anti-CD40-mediated colitis. Data represent mean $\pm \mathrm{s}$.e.m. of five mice per group. (d and $\mathbf{e}$ ) The percentages of interferon- $\gamma\left(\right.$ IFN- $\gamma$ )-producing cells among colonic Thy $1.2^{+}$cell population after injection of WT or re $1^{-/-}$ BMDCs and induction of anti-CD40-mediated colitis in rag1 ${ }^{-1-} \mathrm{rel}^{-1-}$ mice. $\mathbf{d}$ shows representative histogram of two independent experiments. For e, data are mean \pm s.e.m. $\left(n=6\right.$ mice per group). ${ }^{\star \star} P=0.001-0.01$.

pronounced c-Rel expression in $\mathrm{CD}^{+} 8^{+}$macrophages and CD11 ${ }^{+}$DCs, suggesting that APC-derived c-Rel is involved in the intestinal immunopathology in humans (Supplementary Figure S3).

\section{DISCUSSION}

The underlying mechanisms linking CD40 stimulation of APCs with ILC-driven innate intestinal inflammation still remain obscure. In this study, we set out to determine whether the expression of the NF- $\mathrm{KB}$ member $\mathrm{c}-\mathrm{Rel}$ in DCs contributes to the development of anti-CD40-induced colitis. Recently, the role for c-Rel in the development of intestinal inflammation was studied in the chronic model of Helicobacter hepaticusmediated innate colitis as well as in the T-cell transfer model of colitis. ${ }^{26}$ Consistent with previous observation showing the importance of c-Rel in inducing intestinal inflammation, we demonstrate that this transcription factor is also involved in the development of anti-CD40-induced colitis. Here, CD40 stimulation of myeloid cells triggers effector cytokine production by colitogenic Thy ${ }^{+}$ILCs of lymphoid origin thus crucially contributing to the inflammatory loop leading to immunopathology in the gut. ${ }^{13}$

ILCs are a newly described immune cell population, which have been shown to regulate homeostasis and inflammation at mucosal sites. ${ }^{17}$ The experiments presented here show that the
NF- $\kappa \mathrm{B}$ member c-Rel is activated by CD40 ligation. As a consequence, the secretion of IL-12 and IL-23 is induced in colonic APCs, suggesting that the pathogenic effects of c-Rel are probably mediated by IL-12/IL-23-controlled production of proinflammatory cytokines by colitogenic Thy ${ }^{+}$ILCs. Indeed, during the course of anti-CD40 colitis, c-Rel regulated indirectly production of IFN- $\gamma$ by Thy ${ }^{+}$ILCs. While rag1 ${ }^{-/-}$animals developed colonic wasting disease, rag1 ${ }^{-/-}$ $\mathrm{rel}^{-/-}$mice were largely protected from anti-CD40-induced colitis and transfer of WT DCs was capable of inducing intestinal inflammation and IFN- $\gamma$ production in rag1 ${ }^{-/-}$ $\mathrm{rel}^{-\prime-}$ mice.

There is considerable controversy with respect to the colitogenic ILC populations in murine and human ILCs. Although cytokines such as IL-17A and tumour-necrosis factor- $\alpha$ might have an important role, accumulating evidences point to the crucial role for IFN- $\gamma^{+}$ILCs in promoting gut immunopathogenesis in both species. Novel studies suggest that IFN- $\gamma^{+}$ILC subpopulation with colitogenic functions also accumulate in the inflamed intestine of inflammatory bowel disease patients. ${ }^{27,28}$ Notably, we were surprised to observe the lack of involvement of colonic IL-17A in development of antiCD40-mediated-colitis in rag1 ${ }^{-/}$mice, which is in contrary to Helicobacter hepaticus-induced intestinal inflammation. ${ }^{13}$ In agreement with a previous study showing that ILC-derived 
IL-17A was dispensable for anti-CD40-induced innate colitis ${ }^{13}$ and based on our observations that the expression of colonic IL-17A was not significantly changed after anti-CD40 treatment of rag1 ${ }^{-/-}$mice, we suggest that the predominant source of this cytokine are colonic Th17 cells rather than ILCs. Together, the role of IL-17A in intestinal inflammation remains controversial with both, inflammatory and protective effects. $^{29,30}$

In summary, our data provide insights into the mechanisms linking CD40-mediated signaling specificities in DCs and Thy $1^{+}$cell-dependent intestinal inflammation in mice. We identified c-Rel as a crucial factor that controls inflammatory functions of colonic Thy $1^{+}$ILCs. Since the transfer of WT BMDCs into rag1 ${ }^{-/-} \mathrm{rel}^{-/-}$mice induced colonic inflammation and enhanced the production of IFN- $\gamma$ by Thy $1^{+}$ILCs, we propose an indirect mechanism of c-Rel-dependent inflammation in the absence of $\mathrm{T}$ lymphocytes.

\section{METHODS}

Human samples. Inflamed mucosa from colon of patients with Crohn's disease was obtained after intestinal surgery at the Charite University Hospital in Berlin. Non-inflamed colonic samples referred to as "healthy control" were collected after intestinal resection of colorectal cancer, whereby the proximal colon was considered unaffected. All tissue samples were collected after subjects provided informed consent. This study was approved by the local ethics committee of the Charité Universitätsmedizin Berlin, Germany. Immunohistochemistry analysis was carried out on paraffinembedded colonic tissue as described previously. ${ }^{31}$ The following primary antibodies were used for the staining: polyclonal rabbit anti-cRel (Cell Signaling Technology, Danvers, MA), monoclonal mouse anti-CD68, and polyclonal rabbit anti-CD11c (Thermo Scientific, Waltham, MA).

Experimental animals. Mice were kept in specific pathogen-free (SPF) facilities at Max Planck Institute for Infection Biology, Berlin, Germany and Philipps University of Marburg, Marburg, Germany. C57BL/6 mice were purchased from Charles River Laboratories. $\mathrm{rel}^{-/-}$mice were obtained from Hsiou-Chi Liou (Cornell University, $\mathrm{NY}) . \mathrm{rel}^{-/-}, \mathrm{ragl}^{-/-}$and $\mathrm{ragl}^{-1-} \mathrm{rel}^{-/-}$mice were bred on a C57BL/6 background. All animal studies were conducted according to the German animal protection law.

Induction of colitis and histology. Anti-CD40 colitis was induced by IP injecting $100 \mu \mathrm{g}$ of monoclonal anti-CD40 Ab, clone 1C10 (rat IgG2a). The hybridoma producing anti-CD40 Ab, clone $1 \mathrm{C} 10$ was obtained from Manuela Staeber, (Max Planck Institute for Infection Biology, Berlin, Germany). The anti-CD40 Ab was purified using a protein G Sepharose 4 fast Flow column (GE Healthcare, Freiburg, Germany). Rat IgG2a isotype control (clone R35-95, BD Bioscience, Heidelberg, Germany) and phosphate buffered saline injections were used for treatment of age- and sex-matched control mice. Animals were monitored daily throughout the course of the experiment for body weight loss and disease activity. In some experiments, $3 \mathrm{mg}$ of IFN- $\gamma$ blocking $\mathrm{mAb}$ (clone XMG1.2) were co-administered together with $1 \mathrm{C} 10 \mathrm{Ab}$. Thy $1.2^{+}$cells were depleted by injecting $250 \mu \mathrm{g}$ of antiThy $1 \mathrm{Ab}$ (clone $30 \mathrm{H} 12$ ) at days $-1,+1$ and +3 during the induction of anti-CD40-mediated colitis in ragl $1^{-/-}$mice. Histological scores were determined using formalin-fixed tissue sections from proximal colons stained with Hematoxylin and eosin. Colons were prepared for histology 7 days after induction of colitis. The inflammation scoring was performed based on a combination of colonic tissue damage and infiltration of inflammatory cells.
Cytokine secretion by colon cultures and ELISA. Colon pieces of $1 \mathrm{~cm}$ length were cultured in RPMI 1640 medium containing 10\% FCS. Media were supplemented with penicillin/streptomycin (PAA) and gentamycin $\left(50 \mu \mathrm{g} \mathrm{ml}^{-1}\right)$. The supernatants were harvested after $24 \mathrm{~h}$ and cytokine concentrations were measured using ELISA. IL-17A and tumour-necrosis factor- $\alpha$ were detected using OptEIA ELISA kits (BD Bioscience). IL-12 p40 ELISA kit was purchased by eBioscience (Frankfurt, Germany). For detection of IL-23, Ready-Set-Go! ELISA kit (eBioscience) was used with p40 and p19 antibodies ensuring specific measurement of this cytokine.

Generation and transfer of BMDCs. BMDCs were generated from BM of 8- to 12-week-old C57BL/6 and $\mathrm{rel}^{-/-}$mice. BM cells $\left(2 \times 10^{6}\right)$ were seeded on Petri dishes and cultured for 1 week in $10 \mathrm{ml}$ of RPMI medium supplemented with $10 \%$ of $\times 6310$-derived granulocytecolony-stimulating factor. Medium conditioned with $\times 6310$ was replaced on days 3 and 5. On day 7, non-adherent cells were collected and subjected to stimulation with LPS $\left(1 \mu \mathrm{g} \mathrm{ml}^{-1}\right)$, CD $40 \mathrm{~L}$ $\left(1 \mu \mathrm{g} \mathrm{ml}^{-1}\right)$, and anti-CD40 $\left(10 \mu \mathrm{g} \mathrm{ml}^{-1}\right) .1 \mathrm{C} 10$ anti-CD40 mAb was generated at Max Planck Institute for Infection Biology, Berlin and used for all in vitro studies to cross-link CD40 on BMDCs.

For transfer experiments, WT and $\mathrm{rel}^{-/-}$BMDCs were stimulated with LPS $\left(1 \mu \mathrm{g} \mathrm{ml}^{-1}\right)$ for $24 \mathrm{~h}$ and subsequently, $6 \mathrm{~h}$ before the induction of anti-CD40 colitis, $5 \times 10^{6}$ LPS-treated BMDCs were IP injected into rag1 ${ }^{-/-} \mathrm{rel}^{-/-}$mice.

Cell isolation from colonic lamina propria and flow cytometry analysis. Colonic tissues were cut into $1 \mathrm{~cm}$ pieces and washed in $\mathrm{Mg}$ and $\mathrm{Ca}$-free phosphate buffered saline. Colon pieces were shaken in complete media for $30 \mathrm{~min}$ at $37^{\circ} \mathrm{C}$ and subsequently intraepithelial fraction was released by rigorous shaking. The remaining LP cells were further digested with $0.3 \mathrm{mg} \mathrm{ml}^{-1}$ collagenase D and collagenase VIII (Sigma-Aldrich, München, Germany) under constant stirring for $45 \mathrm{~min}$ at $37^{\circ} \mathrm{C}$. The colon cell suspensions were filtered, washed, and layered on $40 \%-70 \%$ percoll gradient. After centrifugation (2000 r.p.m., $30 \mathrm{~min}$ ), the colonic LP cells were collected from the interphase and resuspended in complete colon medium. For flow cytometry analysis, the following Abs were used: anti-Thy1.2 (53-2.1, eBioscience), antiCD40 (HM40-3, eBioscience), anti-CD45 (30-F11, eBioscience), antiGr-1 (RB6-8C5; eBioscience), anti-CD4 (RM4-5, BioLegend, Fell, Germany), anti-CD11c (N418, eBioscience), anti-CD11b (M1/70, BD Pharmigen, Heidelberg, Germany), anti-CD44 (IM7, BD Pharmigen), anti-CD127 (SB/199, BD Pharmigen), anti-c-Kit (ACK2, eBioscience), anti-Sca-1 (D7, eBioscience), anti-B220 (RA3-6B2, BD Pharmigen), and anti-MHC II (M5/114.15.2, eBioscience). For intracellular staining of IFN- $\gamma$ (XMG1.2, eBioscience) and IL-17A (eBio 17B7, eBioscience), colonic LP cells were stimulated for $4 \mathrm{~h}$ with phorbol myristate acetate $\left(50 \mathrm{ng} \mathrm{ml}^{-1}\right)$ and ionomycin $\left(750 \mathrm{ng} \mathrm{ml}^{-1}\right)$ in the presence of Brefeldin A $(10 \mu \mathrm{g} / \mathrm{ml})$.

Western blot analysis. For immunoblotting, BMDCs were stimulated for $2 \mathrm{~h}$ and nuclear and cytoplasmic extract from BMDCs were prepared by lysing the cells in buffer A (10 mM HEPES, pH 7.9, $1.5 \mathrm{~mm}$ $\mathrm{MgCl}_{2}, 10 \mathrm{~mm} \mathrm{KCl}, 20 \mathrm{mM} \mathrm{NaF}, 100 \mu \mathrm{M} \mathrm{Na}_{3} \mathrm{VO}_{4}$, complete protease inhibitor (Roche Molecular Biochemicals, Mannheim, Germany)). After addition of $0.25 \%$ of Nonidet-P40 and incubation for $20 \mathrm{~min}$, cells were centrifuged as supernatants were collected and used as cytoplasmic extracts. For generation of nuclear extracts, pellets were resuspended in the buffer $\mathrm{B}(10 \mathrm{~mm}$ HEPES, $\mathrm{pH} 7.9,420 \mathrm{~mm} \mathrm{NaCl}$, $0.2 \mathrm{~mm}$ EDTA $1.5 \mathrm{~mm} \mathrm{MgCl}_{2}, 10 \mathrm{~mm} \mathrm{KCl}, 20 \mathrm{~mm} \mathrm{NaF}, 100 \mu \mathrm{M} \mathrm{Na}_{3} \mathrm{VO}_{4}$, complete protease inhibitor (Roche Molecular Biochemicals) and incubated for $30 \mathrm{~min}$. After centrifugation (10,000 r.p.m., $10 \mathrm{~min}$ ) to remove cell debris, supernatants containing nuclear proteins were collected and protein concentration was determined. To analyze the nuclear translocation of c-Rel, nitrocellulose membranes were incubated with antibodies against c-Rel, Lamin B (both from Santa Cruz Biotechnology, Heidelberg, Germany), and $\alpha$-tubulin (Sigma-Aldrich). 
EMSA. EMSA assay was performed using chemiluminescent EMSA kit (Thermo Fisher Scientific, Waltham, MA) according to manufacturer instructions. In brief, binding reactions were prepared in $20 \mu \mathrm{l}$ reaction volume containing $10 \mu \mathrm{g}$ of nuclear extracts from stimulated BMDCs and NF- $\kappa \mathrm{B}$ consensus sequence 5'-GCCTGGGAAAGTCCCCTCAACT-biotin- $3^{\prime}$ at room temperature for $20 \mathrm{~min}$. Protein-DNA complexes were resolved on a $4 \%$ native polyacrylamid gel and transferred to a positive nylon membrane. After ultraviolet-crosslinking, the samples were treated with streptavidine-horseradish peroxidase conjugate and incubated with substrate solution.

ELISA-based DNA binding assay. Nuclear extract were prepared as described for immunoblotting and EMSA, and ELISA-based assay for measurement of NF- $\kappa \mathrm{B}$ binding activity to the immobilized consensus sequence $5^{\prime}$-GGGACTTTCC- $3^{\prime}$ was performed using a Trans AM commercial kit (Active Motif, La Hulpe, Belgium). To obtain higher sensitivity, anti-c-Rel Ab sc-70 $\times$ (Santa Cruz Biotechnology) was used.

Chromatin immunoprecipitation. For chromatin immunoprecipitation experiments, BMDCs were generated from C57BL/6 and stimulated with $\alpha$-CD40 $\mathrm{mAb}\left(10 \mu \mathrm{g} \mathrm{ml}^{-1}, 1 \mathrm{C} 10\right)$ for $2 \mathrm{~h}$. A total of $1 \times 10^{6}$ BMDCs were fixed with $1 \%$ formaldehyde, DNA was then fragmented by sonication and immunoprecipitation was performed overnight at $4{ }^{\circ} \mathrm{C}$ with anti-mouse c-Rel IgG Ab (sc-70X, Santa Cruz Biotechnology) or control rabbit IgG. To reverse the crosslinking, the digestion with Proteinase $\mathrm{K}\left(20 \mathrm{mg} \mathrm{ml}^{-1}\right)$ was performed overnight. To determine the c-Rel binding to the $\kappa 1$ site of $p 19$ promotor, the quantitative real-time PCR was performed with the following primer set: $\kappa 1$-forward, 5'-CCCGACCTAGGCCTCTAGC-3'; $\kappa 1$-reverse, 5'-CCTTACAGTGCAGGGACCTT-3'.

Quantitative real-time PCR. After homogenization, total RNA from colonic tissue was extracted using TRIzol reagent (Invitrogen, Carlsbad, CA). Following RNA extraction, the RevertAid First Strand cDNA Synthesis Kit (Thermo Scientific) was used to generate complementary DNA (cDNA) according to the manufacturer's instructions. Quantitative real-time PCR for IL-12p35, IL-12p40, IL-23p19, IL-23R, and Hprt1 was conducted on a StepOnePlus device (Applied Biosystems, Foster City, CA) using the following primers and probes: IL-12p35 primers, forward 5'-TACTAGAGAGACTTCTTCCACAACAAGA G-3' ${ }^{\prime}$, reverse $5^{\prime}$-TCTGGTACATCTTCAAGTCCTCATAGA-3' 5'-AGACGTCTTTGATGATG ACCCTGTGCC-3' ${ }^{\prime}$; IL-12p 40 primers, forward $5^{\prime}$-ATGTGTCCTCAGAAGCTAACCATC- $3^{\prime}$, reverse $5^{\prime}$-CGT GTCACAGGTGAGGTTCACT- ${ }^{\prime}$, probe $5^{\prime}$-GCCATGTGGGAGCTG GAGAAAGACGT-3'; IL-23p19 primers, forward 5' -AGCGGGACAT ATGAATCTACTAAGAGA-3', reverse 5'-GTCCTAGTAGGGAGG TGTGAAGTTG- ${ }^{\prime}$, probe $5^{\prime}$-CCAGTTCTGCTTGCAAAGGATCC GC-3'; IL-23R primers, forward 5'-TCAGTGCTACAATCTTCTTCA GAGGAC-3', reverse 5'-GCCAAGAAGAACATTCCCGA-3', probe $5^{\prime}$-GCCTGCTTCAGGTAATCATCAAGACATT GGACTTTT- $3^{\prime}$; Hprt1 primers, forward $5^{\prime}$-GACCGGTCCCGTCATGC- ${ }^{\prime}$, reverse $5^{\prime}$-T CATAACCTGGTTCATCATCGC- $3^{\prime}$, probe $5^{\prime}$-ACCCGCAGTCCCA GCGTCGTC- $3^{\prime}$. Quantification of $c D N A$ was carried out by normalization to expression of housekeeping gene Hprt1 using the $2^{-\Delta \mathrm{C}(\mathrm{t})}$ method.

Statistical analysis. All data analyses were performed using Student's $t$-test and GraphPad Prism 5 software. $P$-values of $P<0.05$ were considered significant. In all experiments, unpaired Student's $t$-test $P$-values were used $\left({ }^{* *} P<0.001,{ }^{* *} P=0.001-0.01,{ }^{\star} P=0.01-0.05\right)$.

SUPPLEMENTARY MATERIAL is linked to the online version of the paper at http://www.nature.com/mi

\section{ACKNOWLEDGMENTS}

We thank Sonja Dullat (Department of Surgery I, the Charité-Universitätsmedizin Berlin) for technical support. We acknowledge Manuela Staeber (the Max Planck Institute for Infection Biology, Berlin) for antibody preparation. This work was supported by UGMLC and UGMLC/LOEWE grants (MR36 to P. I. Pfefferle and C2P12 to U. Steinhoff), DZL grant (the German Center for Lung Research, Disease Area 22 to P. I. Pfefferle), and by the university grant UKGM 42/2012 MR (U. Steinhoff).

\section{DISCLOSURE}

The authors declared no conflict of interest.

c) 2015 Society for Mucosal Immunology

\section{REFERENCES}

1. Xavier, R.J. \& Podolsky, D.K. Unravelling the pathogenesis of inflammatory bowel disease. Nature 448, 427-434 (2007).

2. Neurath, M.F., Pettersson, S., Meyer zum Buschenfelde, K.H. \& Strober, W. Local administration of antisense phosphorothioate oligonucleotides to the p65 subunit of NF-kappa B abrogates established experimental colitis in mice. Nat. Med. 2, 998-1004 (1996).

3. Neurath, M.F., Becker, C. \& Barbulescu, K. Role of NF-kappaB in immune and inflammatory responses in the gut. Gut 43, 856-860 (1998).

4. Bonizzi, G. \& Karin, M. The two NF-kappaB activation pathways and their role in innate and adaptive immunity. Trends Immunol. 25, 280-288 (2004).

5. Ghosh, S. \& Karin, M. Missing pieces in the NF-kappaB puzzle. Cell 109, S81-S96 (2002).

6. Vallabhapurapu, S. \& Karin, M. Regulation and function of NF-kappaB transcription factors in the immune system. Annu. Rev. Immunol. 27, 693-733 (2009).

7. Sun, S.C. \& Ley, S.C. New insights into NF-kappaB regulation and function. Trends Immunol. 29, 469-478 (2008).

8. Pasparakis, M. Regulation of tissue homeostasis by NF-kappaB signalling: implications for inflammatory diseases. Nat. Rev. Immunol. 9, 778-788 (2009).

9. Beinke, S. \& Ley, S.C. Functions of NF-kappaB1 and NF-kappaB2 in immune cell biology. Biochem. J. 382 (Pt 2), 393-409 (2004).

10. Visekruna, A., Volkov, A. \& Steinhoff, U. A key role for NF-kappaB transcription factor c-Rel in T-lymphocyte-differentiation and effector functions. Clin. Dev. Immunol. 2012, 239368 (2012).

11. Wang, J. et al. Distinct roles of different NF-kappa B subunits in regulating inflammatory and T cell stimulatory gene expression in dendritic cells. J. Immunol. 178, 6777-6788 (2007).

12. Mason, N., Aliberti, J., Caamano, J.C., Liou, H.C. \& Hunter, C.A. Cutting edge: identification of C-Rel-dependent and -independent pathways of $\mathrm{IL}-12$ production during infectious and inflammatory stimuli. J. Immunol. 168, 2590-2594 (2002).

13. Buonocore, S. et al. Innate lymphoid cells drive interleukin-23-dependent innate intestinal pathology. Nature 464, 1371-1375 (2010).

14. Diefenbach, A. \& Vonarbourg, C. Innate lymphocytes induce inflammatory bowel disease. Immunol. Cell Biol. 88, 694-696 (2010).

15. Spits, H. \& Di Santo, J.P. The expanding family of innate lymphoid cells: regulators and effectors of immunity and tissue remodeling. Nat. Immunol. 12, 21-27 (2011)

16. Walker, J.A., Barlow, J.L. \& McKenzie, A.N. Innate lymphoid cells - how did we miss them?. Nat. Rev. Immunol. 13, 75-87 (2013).

17. Spits, H. et al. Innate lymphoid cells-a proposal for uniform nomenclature. Nat. Rev. Immunol. 13, 145-149 (2013).

18. Vonarbourg, C. et al. Regulated expression of nuclear receptor RORgammat confers distinct functional fates to NK cell receptorexpressing RORgammat $(+)$ innate lymphocytes. Immunity 33, 736-751 (2010).

19. Uhlig, H.H. et al. Differential activity of IL-12 and IL-23 in mucosal and systemic innate immune pathology. Immunity 25, 309-318 (2006).

20. Coope, H.J. et al. CD40 regulates the processing of NF-kappaB2 p100 to p52. EMBO J. 21, 5375-5385 (2002).

21. Zarnegar, B., He, J.Q., Oganesyan, G., Hoffmann, A., Baltimore, D. \& Cheng, G. Unique CD40-mediated biological program in B cell activation requires both type 1 and type 2 NF-kappaB activation pathways. Proc. Natl Acad. Sci. USA 101, 8108-8113 (2004).

22. Mise-Omata, S., Kuroda, E., Niikura, J., Yamashita, U., Obata, Y. \& Doi, T.S. A proximal kappaB site in the IL-23 p19 promoter is responsible 
for RelA- and c-Rel-dependent transcription. J. Immunol. 179, 6596-6603 (2007).

23. Carmody, R.J., Ruan, Q., Liou, H.C. \& Chen, Y.H. Essential roles of c-Rel in TLR-induced IL-23 p19 gene expression in dendritic cells. J. Immunol. 178, 186-191 (2007).

24. Chognard, G. et al. the dichotomous pattern of IL-12r and IL-23R expression elucidates the role of IL-12 and IL-23 in inflammation. PloS One 9, e89092 (2014).

25. Klose, C.S. et al. Differentiation of type 1 ILCs from a common progenitor to all helper-like innate lymphoid cell lineages. Cell 157, 340-356 (2014).

26. Wang, Y. et al. c-Rel is essential for the development of innate and T cellinduced colitis. J. Immunol. 180, 8118-8125 (2008).
27. Fuchs, A. et al. Intraepithelial type 1 innate lymphoid cells are a unique subset of IL-12- and IL-15-responsive IFN-gamma-producing cells. Immunity 38, 769-781 (2013).

28. Maloy, K.J. \& Uhlig, H.H. ILC1 populations join the border patrol. Immunity 38, 630-632 (2013).

29. Ito, R. et al. Involvement of IL-17A in the pathogenesis of DSS-induced colitis in mice. Biochem. Biophys. Res. Commun. 377, 12-16 (2008).

30. O'Connor, W. Jr et al. A protective function for interleukin 17A in T cellmediated intestinal inflammation. Nat. Immunol. 10, 603-609 (2009).

31. Hotz, B., Visekruna, A., Buhr, H.J. \& Hotz, H.G. Beyond epithelial to mesenchymal transition: a novel role for the transcription factor Snail in inflammation and wound healing. J. Gastrointest. Surg. 14, 388-397 (2010). 